九州大学学術情報リポジトリ

Kyushu University Institutional Repository

\title{
Reversible Data Hiding Using Maximum and Minimum Filters for Image Interpolation
}

Ono, Genki

Graduate School of Design, Kyushu University

Inoue, Kohe $\mathrm{i}$

Department of Communication Design Science, Faculty of Design, Kyushu University

Urahama, Ki ichi

Department of Communication Design Science, Faculty of Design, Kyushu University

http://hdl. handle. net/2324/1912756

出版情報 : 2017-10-24. IEEE

バージョン:

権利関係 : $\odot 2017$ IEEE. Personal use of this material is permitted. Permission from IEEE must be obtained for all other uses, in any current or future media, including

reprinting/republishing this material for advertising or promotional purposes, creating new collective works, for resale or redistribution to servers or lists, or reuse of any copyrighted component of this work in other works. 


\title{
Reversible Data Hiding Using Maximum and Minimum Filters for Image Interpolation
}

\author{
Genki Ono, Kohei Inoue, Kenji Hara, and Kiichi Urahama \\ Department of Communication Design Science \\ Kyushu University \\ Fukuoka, Japan \\ Email: k-inoue@design.kyushu-u.ac.jp
}

\begin{abstract}
We propose a reversible data hiding method for embedding secret data in a scaled-up image of a cover image, where the scaled-up image is computed using maximum and minimum filters to achieve higher payload than that of conventional methods based on neighbor mean interpolation. Experimental results show that the proposed method outperforms conventional methods in both data-hiding capacity and image quality.
\end{abstract}

\section{INTRODUCTION}

Information hiding is a technology including steganography and watermarking [1]. Among many information hiding techniques, data hiding in images is one of the most promising techniques because of the recent widespread use of digital images. Recently, reversible data hiding methods for digital images have been proposed for embedding a large amount of secret data while keeping a good visual quality of images. Jung and Yoo proposed a reversible data hiding method using image interpolation, and showed that their neighbor mean interpolation (NMI) method achieved higher peak signal-to-noise ratio (PSNR) than conventional interpolation methods [2]. Liu et al. modified Jung's method [2] so that more correlation among neighboring pixels could be used for increasing the embedding capacity [3]. Malik et al. also proposed a modified NMI method, which outperformed Jung's NMI [2] and Chang's enhanced NMI [4] in terms of PSNR [5]. Luo et al. also proposed a reversible watermarking scheme which utilizes the interpolation-error to embed the watermark information [6].

In this paper, we propose a reversible data hiding method using maximum and minimum filters for image interpolation, which can increase the payload of scaled-up images compared with the conventional NMI methods. Experimental results show that the proposed method achieves higher PSNR values and larger amount of embedding capacity.

\section{Proposed Reversible Data Hiding Method}

Let $P=\left[p_{i j}\right]$ be a grayscale cover image of $m \times n$ pixels, where $p_{i j}$ denotes the pixel value at the position $(i, j)$ for $i=1, \ldots, m$ and $j=1, \ldots, n$. Let $Q=\left[q_{k l}\right]$ for $k=1, \ldots, 2 m-1$ and $l=1, \ldots, 2 n-1$ be the scaled-up image of $P$ by interpolation. Then the pixel values in $P$ are partially substituted into $Q$ as $q_{k l}=p_{i j}$ for $k=2(i-1)+1$ and $l=2(j-1)+1$ for $i=1, \ldots, m$ and $j=1, \ldots, n$. That is, when both $k$ and $l$ are odd numbers, $q_{k l}$ has the same value as the corresponding $p_{i j}$.

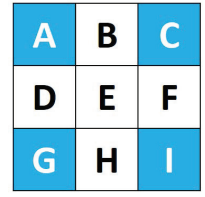

Fig. 1. Illustration of scaled-up image $Q$.

We show an illustration of $Q$ in Fig. 1, where the pixel values in $P$ are substituted for the blue pixels ' $A$ ', 'C', ' $G$ ' and ' $I$ '. For the remaining pixels in $Q$, we calculate the values in two ways by using maximum and minimum filters as follows:

Let $B=\left[b_{k l}\right]$ be a binary image where $b_{k l}=1$ if $q_{k l}$ has a value such that $q_{k l}=p_{i j}, b_{k l}=0$ otherwise. Then one of the interpolated images of $Q$ is given by $Q^{\max }=\left[q_{k l}^{\max }\right]$ with a maximum filter as follows:

$q_{k l}^{\max }= \begin{cases}\max _{-1 \leq v \leq 1}\left\{q_{k+v, l+h} \mid b_{k+v, l+h}=1\right\}, & \text { if } b_{k l}=0, \\ -1 \leq h \leq 1 & \text { otherwise. }\end{cases}$

Another interpolated image of $Q$ is given by $Q^{\min }=\left[q_{k l}^{\min }\right]$ with a minimum filter as follows:

$q_{k l}^{\min }= \begin{cases}\min _{-1 \leq v \leq 1}\left\{q_{k+v, l+h} \mid b_{k+v, l+h}=1\right\}, & \text { if } b_{k l}=0, \\ -1 \leq h \leq 1 & \text { otherwise. }\end{cases}$

From the above two scaled-up images $Q^{\max }$ and $Q^{\min }$, we make a stego image in which secret data are embedded.

\section{A. Embedding Secret Data in Interpolated Image}

Let $s=\left[s_{K}, s_{K-1}, \ldots, s_{2}, s_{1}\right]$ be a secret bit string, whose length is constrained to an integral multiple of 8 bit, i.e., $K \bmod 8=0$, and this constraint will be used in the data extraction stage described in the next subsection. Then we split $s$ into partial bit strings based on the payload of each pixel in the scaled-up images $Q^{\max }$ and $Q^{\min }$. We define the payload at a pixel $(k, l)$ in $Q^{\mathrm{min}}$ by

$$
d_{k l}=q_{k l}^{\max }-q_{k l}^{\min }
$$


from which, the number of bits embedded in this pixel is calculated by

$$
N_{k l}=\left\lfloor\log _{2} d_{k l}\right\rfloor
$$

for $d_{k l}>1$, where $\lfloor\cdot\rfloor$ denotes the floor function. We sort the pixels in ascending order of $d_{k l}$, and embed $N_{k l}$ bit string extracted from $s$ in the pixels with $N_{k l} \geq 1$ in the ascending order. Let $s_{1}=\left[s_{N_{k l}}, s_{N_{k l}-1}, \ldots, s_{2}, s_{1}\right]$ be the first sub bit string in $s$, then we convert $s_{1}$ into the corresponding decimal number $s_{1}^{D}$, and compute the pixel value of the data-embedded image or stego image $\hat{Q}=\left[\hat{q}_{k l}\right]$ as follows:

$$
\hat{q}_{k l}=q_{k l}^{\min }+\boldsymbol{s}_{1}^{D}, \quad \text { if } \boldsymbol{s}_{k l}^{D}<d_{k l},
$$

otherwise we do not embed $\boldsymbol{s}_{1}^{D}$ in the pixel $(k, l)$, and proceed to the next pixel in the sorted order. This procedure is continued until all bit in $s$ is embedded in $\hat{Q}$. After that, there may be some pixels which are not updated by (5) despite $N_{k l} \geq 1$. For such pixels, we substitute $q_{k l}^{\min }$ or $q_{k l}^{\max }$ on the basis of the original or desirable image $F=\left[f_{k l}\right]$ of the same size as $Q$, i.e., $\hat{q}_{k l}=q_{k l}^{\min }$ if $\left|q_{k l}^{\min }-f_{k l}\right|<\left|q_{k l}^{\max }-f_{k l}\right|$, $\hat{q}_{k l}=q_{k l}^{\max }$ otherwise. If the original large image for a given small cover image $P$ does not exist, we can compute an alternative large image from $P$ using image super resolution techniques such as RAISR [7] and SRCNN [8].

\section{B. Extracting Secret Data from Stego Image}

First, we extract the cover image $P$ from $\hat{Q}$ by $p_{i j}=\hat{q}_{k l}$ where $k=2(i-1)+1$ and $l=2(j-1)+1$ for $i=1, \ldots, m$ and $j=1, \ldots, n$. Next, we make the scaled-up images $Q^{\max }$ and $Q^{\text {min }}$ of $P$ by the interpolation method described above. Let $D=\left[d_{k l}\right]=Q^{\max }-Q^{\min }$ be the difference image between $Q^{\max }$ and $Q^{\min }$, then we sort the pixels in ascending order of $d_{k l}$. According to the ascending order, for each pixel with $d_{k l}>1$, we calculate $N_{k l}$ by substituting $d_{k l}$ into (4), and if $N_{k l} \geq 1$ and $\hat{q}_{k l}<q_{k l}^{\max }$, then we calculate $\Delta q=\hat{q}_{k l}-q_{k l}^{\min }$, and convert $\Delta q$ into the bit string of $N_{k l}$ bit. The sequence of $\Delta q$ corresponds to that of the decimal numbers $s_{1}^{D}, s_{2}^{D}, \ldots$ of partial bit strings $s_{1}, s_{2}, \ldots$ We finally concatenate the obtained bit strings, and cut some higher bits to get an integral multiple of 8 bit to reconstruct the embedded secret bit string $s$. This procedure recovers the cover image $P$ and the secret data $s$ completely. Therefore, the proposed method is also reversible as well as the NMI methods.

\section{EXPERIMENTAL RESULTS}

We evaluated the performance of the proposed method with six grayscale images provided by the USC-SIPI Image Database [9]. The number of pixels in each image is $512 \times 512$. Each of those images is denoted by $F$ in the above description of the proposed method. To evaluate the image quality of the stego images generated by the data hiding methods objectively and quantitatively, we scaled down each image $F$ to have a cover image $P$, from which a scaled-up stego image $\hat{Q}$ is computed and compared with $F$.

Fig. 2 shows the payload of each image, where magenta, blue, green and red bars denote Jung's, Liu's, Malik's and

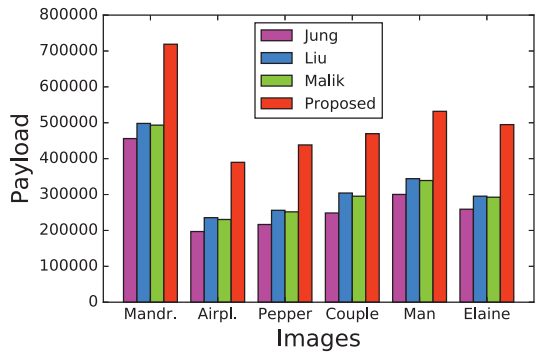

Fig. 2. Payload of Mandrill, Airplane, Pepper, Couple, Man, Elaine images.

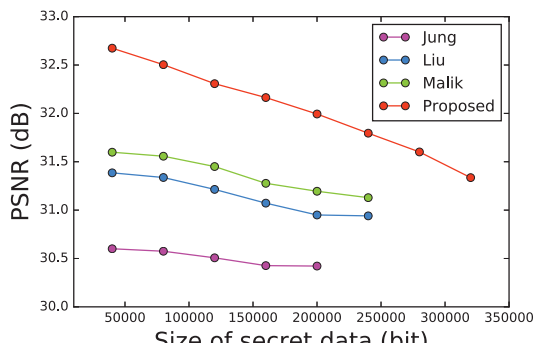

Fig. 3. PSNR.

our methods, respectively. Our method (red bar) achieved the highest payload among the compared methods.

Fig. 3 shows PSNR for the stego images generated from Pepper image and a random secret bit string, where the vertical and horizontal axes denote PSNR and the size of secret data embedded in the stego image. The proposed method (red line) achieved the highest PSNR among the compared methods, and can embed larger amount of secret data than other methods as the red line is elongated to the rightmost point.

\section{ACKNOWLEDGMENT}

This work was supported by JSPS KAKENHI Grant Number JP16H03019.

\section{REFERENCES}

[1] F.A.P. Petitcolas, R.J. Anderson, and M.G. Kuhn, "Information hiding-a survey," Proc. IEEE, vol. 87, no. 7, pp. 1062-1078, 1999.

[2] K.-H. Jung and K.-Y. Yoo, "Data hiding method using image interpolation," Comput. Stand. Interfaces, vol. 31, no. 2, pp. 465-470, Feb. 2009

[3] L. Liu, T. Chen, S. Zhu, W. Hong, and X. Si, "A reversible data hiding method using improved neighbor mean interpolation and random-block division," Information Technology Journal, vol. 13, no. 15, pp. 2374 2384, 2014

[4] Y.-T. Chang, C.-T. Huang, C.-F. Lee, and S.-J. Wang, "Image interpolating based data hiding in conjunction with pixel-shifting of histogram," The Journal of Supercomputing, vol. 66, no. 2, pp. 1093-1110, 2013.

[5] H. K. Verma A. Malik, G. Sikka, "An image interpolation based reversible data hiding scheme using pixel value adjusting feature," Multimed. Tools Appl., 2016.

[6] Lixin Luo, Zhenyong Chen, Ming Chen, Xiao Zeng, and Zhang Xiong, "Reversible image watermarking using interpolation technique," Trans. Info. For. Sec., vol. 5, no. 1, pp. 187-193, Mar. 2010.

[7] P. Milanfar Y. Romano, J. Isidoro, "RAISR: rapid and accurate image super resolution," CoRR, vol. arXiv:1606.01299 [cs.CV], 2016.

[8] C. Dong, C. C. Loy, K. He, and X. Tang, "Image super-resolution using deep convolutional networks," IEEE Trans. Pattern Anal. Mach. Intell., vol. 38, no. 2, pp. 295-307, 2016.

[9] A. Weber, "The usc-sipi image database," http://sipi.usc.edu/database/. 